\title{
New technology to expose core from fiber for optical sensing application
}

\begin{abstract}
For the future optical sensing application, a simple, fast and cost-effective method to achieve exposed core is required for a good mass production and commercialization. Hence, this paper proposes sensing application with exposed core technology using Self-Written Waveguide (SWW) method. The SWW method uses a UV-curable resin and enables fabrication of an optical channel waveguide. SWW is known as an attractive and useful technique in optical interconnection. This SWW is passively aligned between two fibers from the end of the optical fiber under irradiation UV light. This makes the technology is a reliable exposed core technology for sensing application. A SWW with length of 800 micrometers is fabricated from the end face of the multi-mode optical fiber under irradiation with UV light. Experiment is done under several testing materials with different refractive index. The output optical power is decreasing as the refractive indexes of testing materials are increasing. Simulation is also done using ray-tracing method. From these results, it seems possible to apply this SWW using UV-curable resin in sensing application.
\end{abstract}

Keyword: Sensing application; UV-curable resin; Self-written waveguide (SWW); Exposed core; Ray-tracing simulation 